\title{
TERLIPRESSIN THERAPY RESPONSE ASSESSED BY COLOUR DOPPLER IMAGING IN PATIENTS OF HEPATORENAL SYNDROME
}

\author{
Amit Nandan Dhar Dwivedi' ${ }^{1}$,Asif Iqbal2 ${ }^{2}$ Ram Chandra Shukla ${ }^{3}$,Vinod Kumar Dixit ${ }^{4}$, Srishti Sharma 5 , Vairavaganesh R. A6 \\ ${ }^{1}$ Associate Professor, Department of Radiodiagnosis, Institute of Medical Sciences, Banaras Hindu University. \\ 2Junior Resident, Department of Internal Medicine, Institute of Medical Sciences, Banaras Hindu University. \\ ${ }^{3}$ Professor, Department of Radiodiagnosis, Institute of Medical Sciences, Banaras Hindu University. \\ ${ }^{4}$ Professor, Department of Gastroenterology, Institute of Medical Sciences, Banaras Hindu University. \\ 5 Junior Resident, Department of Radiodiagnosis, Institute of Medical Sciences, Banaras Hindu University. \\ 6Junior Resident, Department of Radiodiagnosis, Institute of Medical Sciences, Banaras Hindu University.
}

\section{ABSTRACT}

\section{BACKGROUND}

The resistive index of renal arteries is the most widely used parameter to estimate the arteriolar vascular resistance in HRS. We aimed to evaluate the effects of terlipressin on renal haemodynamics using resistive index of renal interlobar arteries in such patients.

\section{MATERIALS AND METHODS}

An interventional randomised controlled clinical trial was planned with two groups, namely study group and control group. The study enrolled 40 cases of hepatorenal syndrome. These 40 cases were randomly allocated using computer generated random allocation sequence into two groups of size 20 each. The twenty patients of study group received terlipressin therapy, and other twenty comprised the control group. All underwent upper GI endoscopy and Colour Doppler examination. Terlipressin $1 \mathrm{mg} 6$ hourly intravenously was administered to patients in the study group for 2 days after which Doppler studies were repeated to evaluate the effect of terlipressin on resistive index.

Statistical Analysis- Statistical analysis included paired $t$ test for intragroup comparison and unpaired test for intergroup comparison. A ' $p$ ' value of $<0.05$ was considered statistically significant.

Settings and Design- The present study was carried out in Department of Medicine, Department of Radiology Department of Gastroenterology, SS Hospital, BHU Varanasi. A total of 40 cases of clinically suspected and diagnosed cases of hepatorenal syndrome on the basis of criteria defined by International Ascites Club Consensus Workshop in 2007 were taken, among them 20 patients in which terlipressin was used comprised the study group and 20 patients in which terlipressin was not used comprised the control group. Informed consent had been obtained from patients.

\section{RESULTS}

On examination, ascites was detected in all patients in both study and control groups. Majority of chronic liver diseases leading to HRS were related to alcohol followed by hepatitis B in both groups. There was significant reduction in mean resistive indices in interlobar arteries indicating beneficial effect of terlipressin on renal haemodynamics. There was significant decrease in resistive index of interlobar renal arteries in study group as compared to control group, after terlipressin therapy.

\section{CONCLUSION}

This study is first of its kind to utilise colour flow imaging for objective assessment of renal haemodynamics using resistive index as a parameter and study the favourable effect of Terlipressin in patients of hepatorenal syndrome.

\section{KEYWORDS}

Cirrhosis; Complications; Terlipressin; Ultrasound; Liver Failure; Hepatorenal Syndrome.

HOW TO CITE THIS ARTICLE: Dwivedi AND, Iqbal A, Shukla RC, et al. Terlipressin therapy response assessed by colour Doppler imaging in patients of hepatorenal syndrome. J. Evolution Med. Dent. Sci. 2017;6(75):5360-5364, DOI: 10.14260/Jemds/2017/1163

\section{BACKGROUND}

Hepatorenal syndrome (HRS) is described as a "functional" and reversible form of renal failure that occurs in patients with advanced chronic liver disease. The distinctive hallmark feature of HRS is the intense renal vasoconstriction caused by interactions between systemic and portal haemodynamic.

Financial or Other, Competing Interest: None.

Submission 20-07-2017, Peer Review 01-09-2017,

Acceptance 08-09-2017, Published 18-09-2017.

Corresponding Author:

Amit Nandan Dhar Dwivedi,

Department of Radiodiagnosis.

Institute of Medical Sciences Banaras

Hindu University, Varanasi.

E-mail: amitnandan21@yahoo.com

DOI: $10.14260 /$ jemds $/ 2017 / 1163$

\section{(c) (1) $(9)$}

This results in activation of vasoconstrictors and suppression of vasodilators in the renal circulation. Renal failure is a common complication of cirrhosis and is a poor prognostic indicator.

The annual frequency of HRS in cirrhotic patients with ascites is roughly $8 \%$, and in some reports as high as $40 \%$.

Duplex Doppler ultrasonography of the kidneys is a safe easy and non-invasive method to assess blood flow and arterial vascular resistance as a parameter for vasoconstriction. The resistive index of renal arteries is the most widely used parameter to estimate the arteriolar vascular resistance and to evaluate renal haemodynamics. It is regularly used for screening of transplant rejection or to diagnose renal artery stenosis.

Since the main pathophysiology of HRS is splanchnic arterial vasodilatation and renal arterial vasoconstriction that 
causes increase in resistive index (RI) of renal arterial system and the terlipressin (intravenously administered vasopressin 1 receptor agonist) causes selective vasoconstriction of splanchnic arterial vessels, which tends to reverse alterations in renal haemodynamic. In this study, we aim to evaluate effects of terlipressin on renal haemodynamics with the help of resistive index as an indicator of renal interlobar arteries in patients of hepatorenal syndrome.

\section{MATERIALS AND METHODS}

An interventional randomised controlled clinical trial was planned with two groups namely study group and control group.

\section{Sample Size}

Some prior studies have reported normal mean RI values of $0.64 \pm 0.05$ (21 patients), $0.58 \pm 0.05$ (109 kidneys) and $0.62 \pm$ 0.04 (28 patients). In general, most sonographers now consider 0.70 to be the upper threshold of the normal RI in adults. Now considering a difference of $5 \%$ after treatment as compared to before treatment and assuming equal standard deviation of \pm 0.05 for pre and post measurement and further, assuming a moderate coefficient of correlation of 0.60 between pre and post values, the sample size for each group would be 18 taking the level of significance as $5 \%$ and power as $90 \%$. Now further, assuming $10 \%$ loss to follow-up the required sample size will now be 20 in each group.

Thus, the study enrolled 40 cases of hepatorenal syndrome. These 40 cases were randomly allocated using computer generated random allocation sequence into two groups of size 20 each. The twenty patients of study group received terlipressin therapy, and other twenty comprised the control group.

This study was carried out in the collaboration of three departments (Medicine, Radiology and Gastroenterology) of Sir SSL Hospital, Banaras Hindu University, and Varanasi, India during the period of January 2012 to June 2013. A thorough clinical and relevant laboratory evaluation had been performed for enrolment of patients to meet the criteria defined by International Ascites Club Consensus Workshop in 2007. A total of 40 cases of hepatorenal syndrome were enrolled, out them 20 patients received the terlipressin dose (study group) and remaining 20 patients did not receive terlipressin (control group). The study was approved by the review board and ethical committee of Institute of Medical Sciences, Banaras Hindu University, Varanasi. The study protocol conformed to the ethical guidelines of the 1975 Declaration of Helsinki ( $6^{\text {th }}$ revision, 2008). Informed consent was obtained from the patients.

\section{Radiological Evaluation for Resistive Index of Renal Interlobar Arteries}

The patients were instructed to avoid oral intake at least 4 hours before procedure to reduce masking by gas. Patients were excluded if it was not possible to measure resistive index in two different places in each kidney due to massive ascites or masking by gaseous shadows. Colour Doppler examination and calculation of resistive index were performed using a Philips IU-22 ultrasound unit and a 3.5-5 MHz convex probe. Doppler signals were obtained from interlobar arteries along the border of medullary pyramids. These consecutive measurements at the upper middle and lower pole of the organ were averaged, at the same time bipolar length of kidney, s/o kidney size and cortical thickness were also measured. All Doppler examinations were done by single examiner having experience of more than 10 years who was blinded to results of haemodynamic measurement. Resistive index had been determined before and two days after terlipressin therapy.

\section{Terlipressin Therapy}

After baseline radiological evaluation, Terlipressin $1 \mathrm{mg} 6$ hourly intravenously had been administered to patients in the study group for 2 days after which follow-up radiological evaluation had been done by recalculating the resistive index in the interlobar arteries and evaluating the effect of terlipressin on resistive index.

\section{Statistical Analysis}

All values were expressed as Mean \pm SD. Statistical analysis included paired $t$ test for within the group comparison and unpaired t test for between the group comparisons. For basic characteristics and various sign and symptoms, number and percentage were calculated and Chi square test was applied for inter-group comparison of these variables. A 'p' value of $<0.05$ was considered statistically significant.

\section{RESULTS}

The mean age of presentation was 54 years in both study group and controls. In both groups, male population dominated over female. Males constituted $85 \%$ and $90 \%$ in study group and controls respectively without any statistically significant difference $\left(\chi^{2}=0.334^{a}, p=0.846\right)$. In the study group, all patients presented with abdominal distension (100\%), decreased urinary output (100\%) and jaundice (100\%) followed by melaena (65\%), altered sensorium (55\%) and hematemesis (45\%). In the control group, abdominal distension, jaundice and decreased urinary output were universal presenting complaints followed by melaena, haematemesis and altered sensorium $(70 \%, 40 \%$ and $30 \%$ of patients respectively).

On ultrasound examination, ascites was detected in all patients both in study and control groups. Splenomegaly was observed in $90 \%$ in the study group and $80 \%$ of the control group and hepatomegaly was detected in $45 \%$ of the study group and $70 \%$ of the control group.

Majority of chronic liver disease leading to HRS were related to alcohol in both the groups (50\% vs. 45\%) followed by hepatitis B (20\%) in both groups. In the control group, 15\% were related to Hepatitis $\mathrm{C}$ virus as opposed to $10 \%$ in the study group. Cause could not be found in $15 \%$ of the study group while this number was lower in the control group $(10 \%)$. No patient of autoimmune liver disease could be found in this study in any of the two groups. Wilson disease was the cause of chronic liver disease in $5 \%$ in the study group and $10 \%$ in the control group.

\section{Resistive Index Analysis}

The baseline value of mean resistive index value of right and left kidney in upper middle and lower interlobar renal arteries was calculated in both groups (Table 1). In study group, this value for upper middle and lower interlobar arteries were $0.7270 \pm 0.03935,0.7170 \pm 0.03262,0.6995 \pm 0.05021$ respectively while in control group this value for upper middle and lower interlobar arteries were $0.69820 \pm 0.01473,0.6960$ 
$\pm 0.01635,0.7225 \pm 0.01482$ respectively. After administration of terlipressin, resistive index was calculated in the study group (Table 2) and compared with the resistive index of control group (Table 3). The values clearly demonstrate significant reduction in mean resistive indices in all upper, middle and lower interlobar renal arteries which is indicative of significant and beneficial effect of terlipressin on renal haemodynamics in the form of reduction in resistive indices of renal interlobar arteries.

\begin{tabular}{|c|c|c|c|}
\hline Variables & $\begin{array}{c}\text { Study Group } \\
(\mathbf{n = 2 0 )} \\
\text { RI Mean } \pm \text { SD }\end{array}$ & $\begin{array}{c}\text { Control Group } \\
(\mathbf{n = 2 0 )} \\
\text { RI Mean } \pm \text { SD }\end{array}$ & $\begin{array}{c}\text { p- } \\
\text { value }\end{array}$ \\
\hline Interlobar arteries & $0.7270 \pm$ & $0.69820 \pm$ & 0.004 \\
$(\mathrm{R}+\mathrm{L})$ Upper & 0.03935 & 0.01473 & \\
\hline Interlobar arteries & $0.7170 \pm$ & $0.6960 \pm$ & 0.014 \\
$(\mathrm{R}+\mathrm{L})$ Middle & 0.03262 & 0.01635 & \\
\hline Interlobar arteries & $0.6995 \pm$ & $0.7225 \pm$ & 0.057 \\
$(\mathrm{R}+\mathrm{L})$ Lower & 0.05021 & 0.01482 & \\
\hline \multicolumn{2}{|r}{ Table 1. Baseline Resistive Index } \\
in Interlobar Arteries in both Groups \\
\hline
\end{tabular}

\begin{tabular}{|c|c|c|l|}
\hline Variables & $\begin{array}{c}\text { Pretreatment } \\
\text { RI value } \\
\text { (Study Group) } \\
\text { Mean } \pm \text { SD }\end{array}$ & $\begin{array}{c}\text { Post } \\
\text { treatment } \\
\text { RI value } \\
\text { (Study Group) } \\
\text { Mean } \pm \text { SD }\end{array}$ & p-value \\
\hline $\begin{array}{c}\text { Interlobar arteries } \\
\text { (R+L) Upper }\end{array}$ & $0.727 \pm$ & $0.6275 \pm$ & $<0.001$ \\
\hline Interlobar arteries & $0.717 \pm$ & $0.6415 \pm$ & $<0.001$ \\
(R+L) Middle & 0.032620 & 0.0464843 & \\
\hline Interlobar arteries & $0.6995 \pm$ & $0.6340 \pm$ & $<0.001$ \\
(R+L) Lower & 0.05021 & 0.04333 & \\
\hline \multicolumn{3}{|c|}{ Table 2. Effect of Terlipressin on Resistive Index of } \\
Interlobar Arteries in Study Group \\
\hline
\end{tabular}

\begin{tabular}{|c|c|c|c|}
\hline Variables & $\begin{array}{c}\text { R.I. Pre } \\
\text { (Control } \\
\text { Group) } \\
\text { Mean } \pm \text { SD }\end{array}$ & $\begin{array}{c}\text { R.I. Post } \\
\text { (Control } \\
\text { Group) } \\
\text { Mean } \pm \text { SD }\end{array}$ & $\begin{array}{c}\text { p- } \\
\text { value }\end{array}$ \\
\hline $\begin{array}{c}\text { Interlobar } \\
\text { arteries(R+L) } \\
\text { upper }\end{array}$ & $\begin{array}{c}0.6980 \pm \\
0.01473\end{array}$ & $\begin{array}{c}0.6990 \pm \\
0.01372\end{array}$ & 0.541 \\
\hline $\begin{array}{c}\text { Interlobar } \\
\text { arteries (R+L) } \\
\text { middle }\end{array}$ & $0.6960 \pm$ & $0.69600 \pm$ & 1.000 \\
\hline \multicolumn{2}{|c|}{$\begin{array}{c}\text { Interlobar } \\
\text { arteries (R+L) } \\
\text { lower }\end{array}$} & 0.01060 & $0.7225 \pm$ \\
\hline \multicolumn{2}{|c|}{ Table 3. Resistive Index in Control Group who did not } \\
\multicolumn{2}{|c|}{ Receive Terlipressin Therapy } \\
\hline
\end{tabular}

\section{DISCUSSION}

Hepatorenal syndrome (HRS) is a reversible form of functional renal failure that occurs predominantly with advanced liver disease and fulminant liver failure.[1] Despite advancing research in HRS, its aetiology and medical therapy has not been fully resolved. HRS encompasses 2 distinct types. Type 1 HRS often manifests itself rapidly; without appropriate treatment the mean survival time is approximately 2 weeks.[2] The core feature of pathogenesis of HRS is peripheral arterial vasodilatation, particularly in the splanchnic vasculature. ${ }^{[3,4]}$ This develops with advanced liver cirrhosis, which causes increased resistance to blood flow with high portal pressure. In turn, to reduce the pressure within the hepatic portal system, locally acting vasoactive substances are released that cause vasodilatation of the splanchnic vasculature. ${ }^{[5]}$ These compensatory mechanisms with time become detrimental and result in sustained severe intrarenal arterial vasoconstriction with progressive physiological renal failure. The pooling of blood in the splanchnic vascular bed with the associated hypoperfusion of the kidneys and the ensuing intrarenal arterial vasoconstriction forms the basis for the development of HRS. In the present study, majority of patients were alcohol related in both groups ( $50 \%$ vs. $45 \%$ ) followed by hepatitis B (20\% each) related in both the groups. (Table 2) Thomas $R$ Frieden et al in his study found the aetiology of CLD as follows: both alcohol and hepatitis C infection (46\%); alcohol abuse alone, (29\%); HCV related alone (12\%); both alcohol abuse and chronic hepatitis B virus infection (6\%).[6] Mendez Sanchez in his study found the various aetiologies of liver cirrhosis as follows: alcohol $39.5 \%$, HCV $36.6 \%$, cryptogenic $10.4 \%$, HBV in 5\%.[7] In a study done by Hsu YN et al in elderly Asian population, it was found that $44.9 \%$ had chronic hepatitis $C$ and $19 \%$ had had chronic hepatitis B. ${ }^{[8]}$ In a study done in Maharashtra by Amrapurakar et al, he found following aetiologies: HBV related $40.5 \%$, alcohol related $39 \%$ and $\mathrm{HCV}$ related $17 \%$ and rest (3.5\%) to be cryptogenic which is very similar to our results.[9] Duplex ultrasonography is a widely used safe non-invasive method to assess vascular patency and blood flow in many sites. Duplex Doppler can be used to assess vascular resistance in the small intraparenchymal vessels through simple analysis of Doppler waveform by a parameter termed the resistive index (RI). An elevated renal RI has been observed in various conditions associated with elevated renal vascular resistance such as kidney obstruction, acute tubular necrosis and haemolytic uremic syndrome and should be detected in liver disease related functional kidney failure. Previous data show that RI values decrease from renal hilum to the cortex. RI values measured in the interlobar-arcuate arteries are expected to show the most consistent results. The correlation between increased RI and azotaemia seems to confirm the role of vasoconstriction in the pathogenesis of cirrhotic kidney disease. Doppler ultrasound measurement of the RI is a useful index to quantify renovascular resistance in cirrhotic patients before HRS develops. HRS has very poor prognosis with spontaneous recovery being unlikely.[10] Treatment of HRS can be divided into medical and surgical. The rationale of the use of vasoconstrictors in the treatment of type 1 HRS is to counteract the splanchnic arterial vasodilation in order to improve effective circulating volume and reduce portal pressure. Among vasoconstrictors, to this day, terlipressin is the most widely used in the treatment of type 1 HRS.[11] In most studies, terlipressin has been used together with albumin starting with a priming dose of $1 \mathrm{~g} / \mathrm{kg}$ BW followed by 20-40 g/day, monitoring central venous pressure. In two studies in which terlipressin was given also alone, reversal of renal failure was lower than in the studies in which terlipressin was associated with albumin. ${ }^{[12]}$ In this study, we found a significant increase in the renal resistive index of interlobar arteries among both groups. Maroto et al demonstrated that RI is significantly higher in decompensated cirrhotic patients with ascites than in compensated cirrhotic patients and that the RI of compensated cirrhotic patients is higher than in the controls. The relative risk of developing HRS 
in patients with an $\mathrm{RI}=0.70$ was high. $\mathrm{RI}$ is a useful indicator in patients with cirrhosis and ascites for the diagnosis and prognosis of HRS.[13] In Italy, Sacerdoti et al reported that the Pulsatile Index and Resistive Index were significantly higher in non-ascitic cirrhotic patients than in control patients. The Pulsatile Index and Resistive Index were significantly higher in Child-Turcotte-Pugh class B and C patients than in class A patients. This non-invasive method may be applied to pathophysiological and clinical studies of the renal functional impairment in cirrhosis.[14] Hadengue et al carried out a double-blind, crossover, randomised study in 9 patients with type $1 \mathrm{HRS}$. The patients received terlipressin $(2 \mathrm{mg} /$ days for 2 days) and a placebo for 2 days in a randomised order. Terlipressin administration significantly increased creatinine clearance and urine output, but did not significantly change urinary sodium concentration. Urinary sodium excretion was not significantly different after placebo administration or terlipressin administration.[15] The study by Solanki et al was a randomised, controlled, single-blind trial. They assigned 24 consecutive patients with HRS to treatment with terlipressin 1 mg IV at 12 -hour intervals (group $A, n=12$ ) or placebo at 12 hour intervals (group $B, n=12$ ). The end-point of the study was improvement in renal function defined as reversal of HRS and survival at 15 days. Terlipressin administration was shown to be associated with an improvement in parameters of renal function, mean arterial blood pressure and importantly reversal of HRS in 5 of 12 patients of group A.[16] Robertson M et al[17] reviewed outcomes of 69 patients treated with terlipressin between 2001 and 2005. Forty-one (59.4\%) patients responded to terlipressin. Twenty-one (30.4\%) patients survived; 17 (81\%) had type 1 HRS while 4 (19\%) had type 2 HRS $(\mathrm{P}=0.27)$. Terlipressin associated with albumin therapy was associated with marked improvement in renal function, reversal of HRS and improvement in circulatory function with an increase in mean arterial blood pressure.[1820] Several meta-analyses have been conducted to determine the effect of terlipressin in HRS with regard to the duration of treatment.[21,22] Fabrizi et al[23,24] showed in their metaanalysis that discontinuation of terlipressin therapy was associated with a significant increase in the number of relapses. Furthermore, terlipressin was more effective in reversing HRS than placebo without apparent impact of terlipressin on survival in HRS patients. This may again suggest the need for large clinical trials addressing the impact of terlipressin in HRS patient survival. Interestingly, administration of albumin with terlipressin showed a reduction in mortality in type 1 HRS. Therefore, the current evidence from abovementioned trials suggests that terlipressin can have a potential benefit in treating HRS and that an improvement in survival. Terlipressin has few adverse side effects, which allows patients to continue with treatment in order to achieve desirable effects.

\section{CONCLUSION}

In our study, we evaluated the effect of terlipressin therapy on renal haemodynamics in patients of hepatorenal syndrome which showed significant decrease in resistive indices of renal interlobar arteries. Hence, we conclude that terlipressin causes significant decrease in renal vasoconstriction, which is a key feature in pathophysiology of renal injury observed in patients of hepatorenal syndrome, and therefore, terlipressin based therapy is the sheet anchor in medical management of hepatorenal syndrome.

There was significant decrease in resistive index of interlobar renal arteries in study group as compared to control group, after terlipressin therapy, confirming previous studies and indicating the fact that terlipressin causes significant reduction in renal vasoconstriction as reflected by decrease in resistive indices of interlobar renal arteries.

There was no significant change in cortical thickness of kidney in both groups elaborating the fact that this parameter is indicative of chronic disease process and not altered by short-term terlipressin therapy.

This study is first of its kind to utilise colour flow imaging for objective assessment of renal haemodynamics using resistive index as a parameter and study favourable effect of Terlipressin in patients of hepatorenal syndrome.

\section{REFERENCES}

[1] Arroyo V, Ginès P, Gerbes AL, et al. Definition and diagnostic criteria of refractory ascites and hepatorenal syndrome in cirrhosis. International Ascites Club. Hepatology 1996;23(1):164-76.

[2] Ginès $P$, Guevara $M$, Arroyo $V$, et al. Hepatorenal syndrome. Lancet 2003;362(9398):1819-27.

[3] Simonson MS, Rooney A, Herman WH. Expression and differential regulation of Id1, a dominant negative regulator of basic helix-loop-helix transcription factors, in glomerular mesangial cells. Nucleic Acids Res 1993;21(24):5767-74.

[4] Gluud LL, Kjaer MS, Christensen E. Terlipressin for hepatorenal syndrome. Cochrane Database Syst Rev 2006;(4):CD005162.

[5] Wadei HM, Mai ML, Ahsan N, et al. Hepatorenal syndrome: pathophysiology and management. Clin J Am Soc Nephrol 2006;1(5):1066-79.

[6] Frieden TR, Ozick L, McCord C, et al. Chronic liver disease in central Harlem: the role of alcohol and viral hepatitis. Hepatology 1999;29(3):883-8.

[7] Méndez-Sánchez N, Aguilar-Ramírez JR, Reyes A, et al. Etiology of liver cirrhosis in Mexico. Ann Hepatol 2004;3(1):30-3.

[8] Hsu YN, Pan CQ, Abbasi A, et al. Clinical presentation and disease phases of chronic Hepatitis B using conventional versus modified ALT criteria in Asian Americans. Dig Dis Sci 2014;59(4):865-71.

[9] Amarapurkar DN, Kumar A, Parikh SS, et al. Hepatitis C virus infection in chronic liver disease in Bombay. Indian J Gastroenterol 1992;11(4):162-3.

[10] Narahara Y, Kanazawa H, Taki Y, et al. Effects of terlipressin on systemic, hepatic and renal hemodynamics in patients with cirrhosis. J Gastroenterol Hepatol 2009;24(11):1791-7.

[11] Halimi C, Bonnard P, Bernard B, et al. Effect of terlipressin (Glypressin) on hepatorenal syndrome in cirrhotic patients: results of a multicentre pilot study. Eur J Gastroenterol Hepatol 2002;14(2):153-8.

[12] Uriz J, Ginès $P$, Cárdenas $A$, et al. Terlipressin plus albumin infusion: an effective and safe therapy of hepatorenal syndrome. J Hepatol 2000;33(1):43-8. 
[13] Maroto A, Ginès A, Saló J, et al. Diagnosis of functional renal failure of cirrhosis with Doppler sonography: prognostic value of resistive index. Hepatology 1994;20(4 Pt 1):839-44.

[14] Sacerdoti D, Bolognesi M, Merkel C, et al. Renal vasoconstriction in cirrhosis evaluated by duplexDoppler ultrasonography. Hepatology 1993;17(2):21924.

[15] Hadengue A, Gadano A, Moreau R, et al. Beneficial effects of the 2-day administration of terlipressin in patients with cirrhosis and hepatorenal syndrome. J Hepatol 1998;29(4):565-70.

[16] Solanki P, Chawla A, Garg R, et al. Beneficial effects of terlipressin in hepatorenal syndrome: a prospective, randomized placebo- controlled clinical trial. J Gastroenterol Hepatol 2003;18(2):152-6.

[17] Robertson M, Majumdar A, Garrett K, et al. Continuous outpatient terlipressin infusion for hepatorenal syndrome as a bridge to successful liver transplantation. Hepatology 2014;60(6):2125-6.

[18] Sanyal AJ, Boyer T, Garcia-Tsao G, et al. A randomized, prospective, double blind, placebo-controlled trial of terlipressin for type 1 hepatorenal syndrome. Gastroenterology 2008;134(5):1360-8.
[19] Martín-Llahí M, Pépin MN, Guevara M, et al. Terlipressin and albumin vs. albumin in patients with cirrhosis and hepatorenal syndrome: a randomized study. Gastroenterology 2008;134(5):1352-9.

[20] Neri S, Pulvirenti D, Malaguarnera M, et al. Terlipressin and albumin in patients with cirrhosis and type I hepatorenal syndrome. Dig Dis Sci 2008;53(3):830-5.

[21] Dobre M, Demirjian S, Sehgal AR, et al. Terlipressin in hepatorenal syndrome: a systematic review and metaanalysis. Int Urol Nephrol 2011;43(1):175-84.

[22] Sagi SV, Mittal S, Kasturi KS, et al. Terlipressin therapy for reversal of type 1 hepatorenal syndrome: a metaanalysis of randomized controlled trials. J Gastroenterol Hepatol 2010;25(5):880-5.

[23] Fabrizi F, Dixit V, Martin P. Meta-analysis: terlipressin therapy for the hepatorenal syndrome. Aliment Pharmacol Ther 2006;24(6):935-44.

[24] Fabrizi F, Dixit V, Messa P, et al. Terlipressin for hepatorenal syndrome: a meta-analysis of randomized trials. Int J Artif Organs 2009;32(3):133-40. 